\title{
A cytokine-mediated link between innate immunity, inflammation, and cancer
}

\author{
Wan-Wan Lin ${ }^{1}$ and Michael Karin²
}

1Department of Pharmacology, College of Medicine, National Taiwan University, Taipei, Republic of China. ${ }^{2}$ Laboratory of Gene Regulation and Signal Transduction, Department of Pharmacology and Cancer Center, School of Medicine, UCSD, La Jolla, California, USA.

\begin{abstract}
It has been established that cancer can be promoted and/or exacerbated by inflammation and infections. Indeed, chronic inflammation orchestrates a tumor-supporting microenvironment that is an indispensable participant in the neoplastic process. The mechanisms that link infection, innate immunity, inflammation, and cancer are being unraveled at a fast pace. Important components in this linkage are the cytokines produced by activated innate immune cells that stimulate tumor growth and progression. In addition, soluble mediators produced by cancer cells recruit and activate inflammatory cells, which further stimulate tumor progression. However, inflammatory cells also produce cytokines that can limit tumor growth. Here we provide an overview of the current understanding of the role of inflammation-induced cytokines in tumor initiation, promotion, and progression.
\end{abstract}

\section{Mechanisms that link inflammation and cancer}

Cancer is a hyperproliferative disorder that involves morphological cellular transformation, dysregulation of apoptosis, uncontrolled cellular proliferation, invasion, angiogenesis, and metastasis (1). Clinical and epidemiologic studies have suggested a strong association between chronic infection, inflammation, and cancer (2-6). For example, there are strong associations between alcohol abuse, which leads to inflammation of the liver and pancreas, and cancers of these organs. Cigarette smoking, asbestos exposure, and silica exposure are each associated with inflammation of the lung and lung carcinoma; inflammatory bowel disease (IBD) is associated with colon cancer; infection with Helicobacter pylori is associated with gastric carcinoma; chronic viral hepatitis is associated with liver cancer; infection with Schistosoma spp. is associated with bladder and colon carcinoma; infection with some strains of HPV is associated with cervical cancer; and infection with EBV is associated with Burkitt lymphoma and nasopharyngeal carcinoma. Such observations suggest that chronic inflammation is involved in tumor initiation (the process by which normal cells are genetically altered so that they become malignant), promotion (the process by which small clusters of malignant cells are stimulated to grow), and progression (the process by which the growing tumor becomes more aggressive) (2-7). Recent data from mouse models of human cancer have established that inflammation, which orchestrates the tumor microenvironment, is a critical component of both tumor promotion and tumor progression (8-10).

The inflammatory milieu is occupied by cells such as resident and recruited macrophages, DCs, T cells, and NK cells (11). Among these, tumor-associated macrophages (TAMs) and $\mathrm{T}$ cells are frequently the prominent leukocytes present in a tumor $(12,13)$. The infiltrated immune cells can exert rather paradoxical effects during cancer development (Figure 1) (7). Most current

Nonstandard abbreviations used: CAC, colitis-associated colon cancer; HCC, hepatocellular carcinoma; IBD, inflammatory bowel disease; IKK, inhibitor of NF-кB kinase; MM, multiple myeloma; NOD, nucleotide-binding oligomerization domain; NSCLC; non-small cell lung carcinoma; PAMP, pathogen-associated molecular pattern; PRR, pattern recognition receptor; TAM, tumor-associated macrophage; TRAIL, TNF-related apoptosis-inducing ligand.

Conflict of interest: The authors have declared that no conflict of interest exists. Citation for this article: J. Clin. Invest. 117:1175-1183 (2007). doi:10.1172/JCI31537. data support the notion that acute inflammation triggered by tumor-infiltrating host leukocytes does not exert normal immunoprotective mechanisms that lead to eradication of the evolving cancer (antitumor immunity). Instead, excessively and chronically produced proinflammatory mediators are thought to contribute to tumor promotion and progression $(2,8,14,15)$. In the tumor microenvironment, there is a delicate balance between antitumor immunity and tumor-originated proinflammatory activity, which weakens antitumor immunity $(14,16)$. These activities depend on different mediators that are released by host inflammatory cells, cancer cells, and other types of tumor-associated host cells (such as fibroblasts and endothelial cells). When host-mediated antitumor activity is weaker than tumor-mediated immunosuppressive activity, tumor cells undergo immune escape and grow rapidly (17). By contrast, when host-mediated antitumor immunity is stronger than tumor-mediated immunosuppressive activity, tumor cells are eliminated (17). The net outcome of a persistent inflammatory microenvironment is enhanced tumor promotion, accelerated tumor progression, invasion of the surrounding tissues, angiogenesis, and often metastasis $(2,6,9,10)$.

A key molecular link between inflammation and tumor promotion and progression is provided by the inhibitor of NF- $\kappa \mathrm{B}$ kinase/

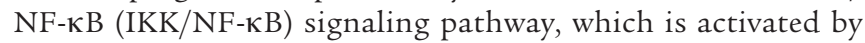
many proinflammatory cytokines $(9,10)$. NF- $\kappa B$ is a transcription factor that regulates the expression of many genes whose products can suppress tumor cell death; stimulate tumor cell cycle progression; enhance epithelial-to-mesenchymal transition, which has an important role in tumor invasiveness; and provide newly emerging tumors with an inflammatory microenvironment that supports their progression, invasion of surrounding tissues, angiogenesis, and metastasis $(6,10,18-20)$.

\section{Infection, innate immunity, and tumorigenesis}

Chronic inflammation caused by persistent infection with a parasite, bacterium, or virus is a major driving force in tumor development $(2,9)$ (Figure 1). It was noted that bacterial infection following the surgical removal of primary tumors can promote rapid growth of metastases in mice (21) and humans (22). Infectious organisms trigger inflammation through activation of receptors that recognize pathogen-associated molecular patterns 


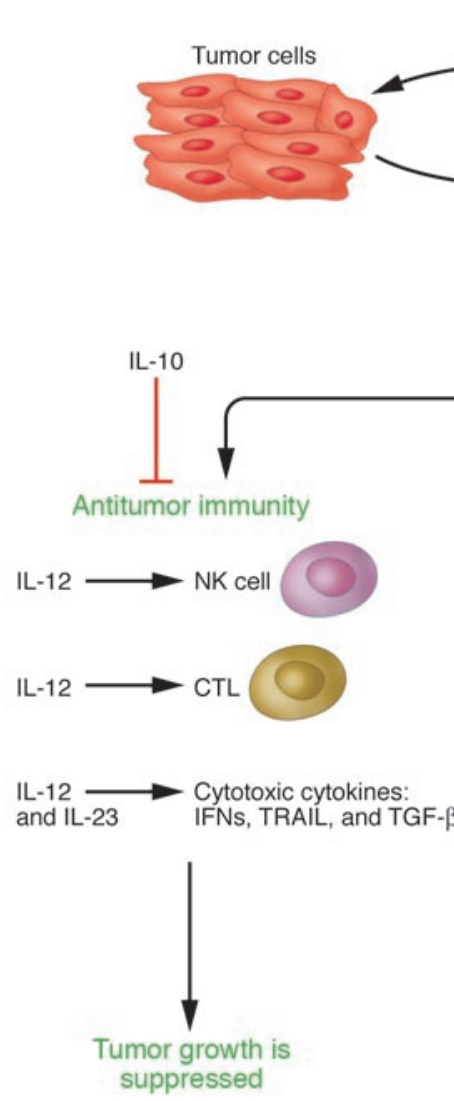

\section{Figure 1}

The diagram shows two outcomes of interactions between tumor cells and infiltrating inflammatory and/or immune cells in the tumor microenvironment. Cytokines secreted by tumor and inflammatory/immune cells can either promote tumor development and tumor cell survival or exert antitumor effects. Chronic inflammation develops through the action of various inflammatory mediators, including TNF- $\alpha$, IL-6, and IL-17, leading to eradication of antitumor immunity and accelerated tumor progression. However, TRAIL, through direct induction of tumor cell apoptosis, IL-10, through antiinflammatory effects, and IL-12, through activation of CTLs and NK cells and expression of cytotoxic mediators, can lead to tumor suppression. The multiple actions of TGF- $\beta$ (cytotoxic in colon cancer cells, and having both positive and negative effects on the tumor microenvironment) and IL-23 (see Figure 3) explain their dual roles in tumor development.
(PAMPs), such as cell wall components and nucleic acids (23). At least four families of mammalian innate immune receptors that recognize PAMPs have been identified; these are known as pattern recognition receptors (PRRs) and include TLRs, nucleotide-binding oligomerization domain-like (NOD-like) receptors (NLRs), C-type lectin receptors (CLRs), and triggering receptors expressed on myeloid cells (TREMs) (24-27). The interaction between PAMPs and PRRs results in the activation of inflammatory cells and initiation of host responses whose major purpose is to eliminate and kill invading organisms (9). However, inadequate pathogen eradication, prolonged inflammatory signaling, and defects in antiinflammatory mechanisms can all lead to chronic inflammation and benefit tumor development (28).

In addition to epidemiological data linking chronic infections to increased cancer risk $(2,3,29)$, genetic links between PRRs and cancer also exist. Polymorphisms in a gene cluster encoding TLR6 and TLR10 have been linked to an increased risk of prostate cancer (30), and mutations in the NOD2 locus have been linked to an increased risk for developing Crohn disease (31), an IBD associated with a modestly increased risk of developing colorectal cancer (32). These mutations in NOD2 have been suggested to provide a gain-of-function that results in increased IL- $1 \beta$ production, which makes the environment more proinflammatory (33). Interestingly, polymorphisms in the promoter region of the gene encoding IL-1 $\beta$ and the gene encoding the IL-1 receptor antagonist (IL-1RA) have also been linked to an increased risk of developing cancer, in particular gastric cancer $(34,35)$. IL-1 is abundant at tumor sites, where it can stimulate the growth and invasiveness of malignant cells (36). Inhibition of IL-1 function using the naturally occurring inhibitor of IL-1, IL-1RA, might be useful for the treatment of cancer (37).
Activation of TLR signaling can enhance tumor development through various mechanisms. In a mouse model of transplanted metastatic cancers, activation of TLR4 by intraperitoneal injection of bacterial LPS stimulated the growth of lung metastases $(21,38,39)$. TLR4 activation of host macrophages resulted in the production of several different inflammatory cytokines that influenced tumor growth. TNF- $\alpha$ was identified as the major host-produced factor that enhances the growth of lung metastases in this mouse model, in part through activation of NF- $\kappa B$ in the tumor cells (39). However, TLR4 signaling also induced the production of IFNs, cytokines that have antitumor effects (39). In this particular case, IFNs were found to stimulate production of the TNF superfamily member TNF-related apoptosis-inducing ligand (TRAIL) (39). TRAIL is a potent inducer of tumor cell death (40), but in this mouse model of transplanted metastatic cancer, its tumoricidal activity was evident only upon inhibition of NF- $\kappa$ B activity in the tumor cells (39). These results illustrate that activation of innate immunity results in the production of different cytokines with opposing activities (Figure 2) - TNF- $\alpha$ stimulated tumor cell growth and survival, whereas TRAIL induced tumor cell death, leading to tumor regression. However, only by inhibiting NF- $\mathrm{KB}$ activation in the tumor cells was the balance shifted from stimulation of tumor growth by TNF- $\alpha$ to enhanced tumor cell killing by TRAIL (39).

TLR expression and function are not restricted to innate immune cells and can directly affect the tumor cell. For example, multiple myeloma (MM) cells frequently express multiple TLRs and can thereby sense the presence of microorganisms $(41,42)$. Indeed, ligands for both TLR7 and TLR9 have been shown to stimulate the growth of MM cells and to protect these cells from 


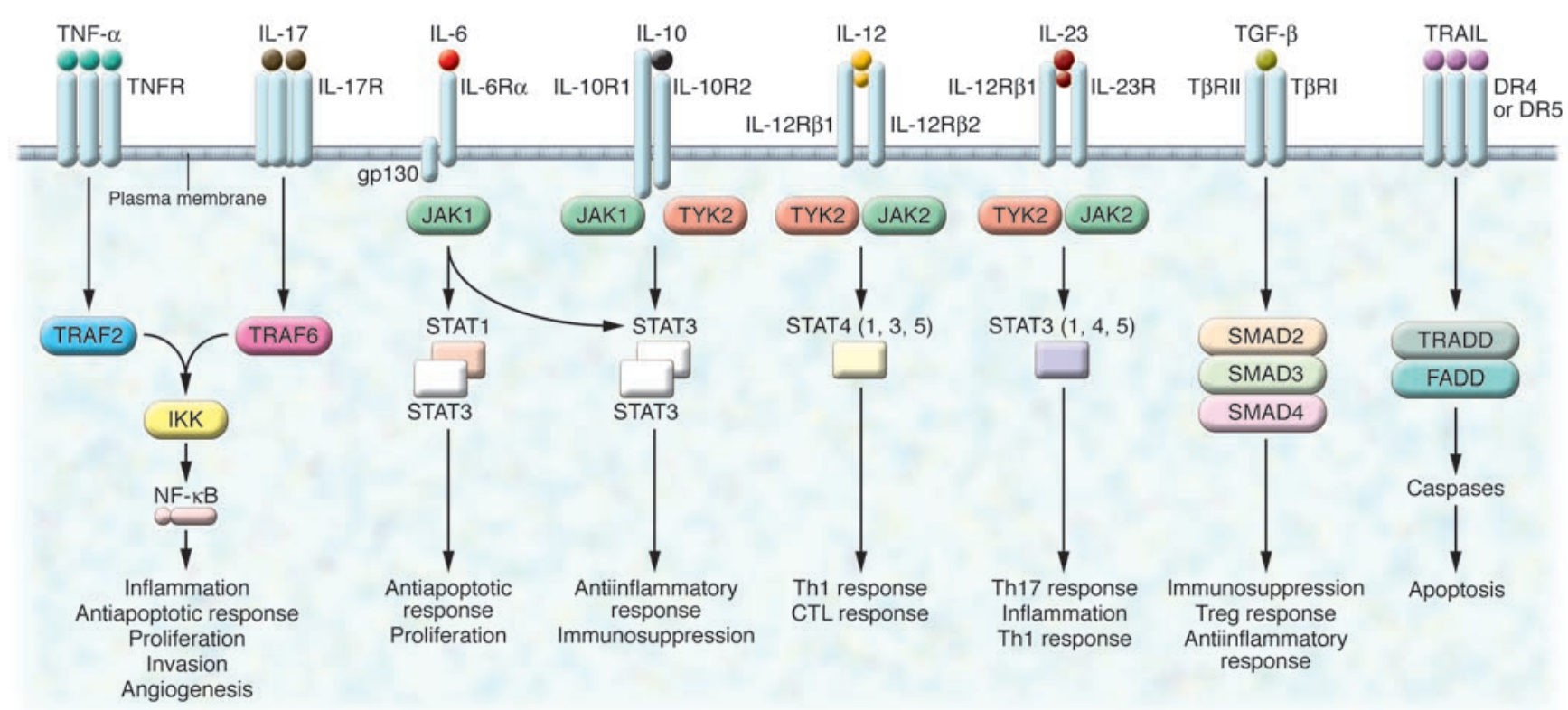

Figure 2

Signal transduction pathways and major biological responses of inflammation-modulating cytokines in cancer. The signaling pathways shown can control tumor development through a direct effect on tumor cells (e.g., NF-кB, STAT3, and caspases) and/or an indirect effect on immune and endothelial cells (e.g., NF-KB, STAT3, STAT4, and SMAD). DR4, death receptor 4; FADD, Fas-associated death domain; gp130, glycoprotein 130; TRADD, TNF receptor-associated death domain protein; TRAF2, TNF receptor-associated factor 2; TYK2, tyrosine kinase 2.

apoptosis (41). This finding could explain why recurrent infections promote the progression of MM (41).

This Review focuses on the role of various cytokines produced by innate immune cells on tumor development and progression. The cytokines to be discussed include TNF- $\alpha$, TRAIL, IL-6, IL-17, IL-12, IL-23, IL-10, and TGF- $\beta$ (Figure 2). As mentioned above, these cytokines can promote and/or inhibit tumor development. The involvement of chemokines in chronic inflammation-associated tumor progression will not be covered in this Review.

\section{TNF- $\alpha$}

The critical role of TNF- $\alpha$ in chronic inflammatory diseases is well established (43), and its tumor-promoting effects have been demonstrated (44). TNF- $\alpha$ produced by tumor cells or inflammatory cells in the tumor microenvironment can promote tumor cell survival through the induction of genes encoding NF-KB-dependent antiapoptotic molecules (39) (Figures 2 and 3). In asbestos-induced human malignant mesothelioma, macrophages phagocytose asbestos and then release TNF- $\alpha$. This TNF- $\alpha$ promotes cell survival and thereby reduces asbestos-induced cytotoxicity, increasing the pool of asbestos-damaged mesothelial cells that are susceptible to malignant transformation (45). TNF- $\alpha$ has also been proposed to contribute to tumor initiation by stimulating the production of genotoxic molecules, that is, molecules that can lead to DNA damage and mutations, such as NO and ROS (4). Genetic polymorphisms that enhance TNF- $\alpha$ production are associated with increased risk of MM, bladder cancer, hepatocellular carcinoma (HCC), gastric cancer, and breast cancer, as well as poor prognosis in various hematological malignancies (44). Other actions of TNF- $\alpha$ that might enhance tumor progression, as opposed to tumor initiation, include promotion of angiogenesis and metastasis, as well as impairment of immune surveillance by strongly suppressing many $\mathrm{T}$ cell responses and the cytotoxic activity of activated macrophages (46).
Studies have suggested a role for keratinocyte-produced TNF- $\alpha$ in mouse models of skin carcinogenesis $(47,48)$. Skin cancer induction by administration of the carcinogen 7,12-dimethylbenz[a]anthracene (DMBA) and tumor promoters results in much higher tumor incidence in TNF- $\alpha$-sufficient mice than TNF- $\alpha$-deficient mice (47). Reduced tumorigenesis was also found in mice deficient in both the TNF- $\alpha$ receptors TNFR1 and TNFR2 (48). A tumor-promoting role for TNF- $\alpha$ has also been found in cholestatic liver cancer, which develops as a result of chronic liver inflammation in mice lacking the drug and phospholipid transporter MDR2 (49). Treatment with TNF- $\alpha-$ specific neutralizing antibody during the tumor promotion stage resulted in apoptosis of transformed hepatocytes and a failure to progress to HCC. An important role for TNF- $\alpha$ in the promotion phase of HCC has also been suggested (50). In that study, TNF- $\alpha$ expression was upregulated during liver stem cell proliferation induced by a choline-deficient and ethioninesupplemented diet. Liver stem cell proliferation and tumorigenesis were found to depend on TNFR1. Yet another study has indicated that TNF- $\alpha$ signaling is crucial for promoting liver metastasis of a colon adenocarcinoma line (51). In another tumor transplantation model of cancer, LPS administration to tumor-bearing mice induced production of TNF- $\alpha$ and stimulated metastatic tumor growth in the lung (39). Taken together, proinflammatory TNF- $\alpha$ released by host and tumor cells is an important factor involved in initiation, proliferation, angiogenesis, and metastasis of various types of cancers.

\section{TRAIL}

The TNF superfamily member TRAIL can bind five different receptors, two of which, death receptor 4 (DR4) and DR5, have cytoplasmic death domains that deliver caspase-dependent apoptotic signals to the cell on which they are expressed (52). 


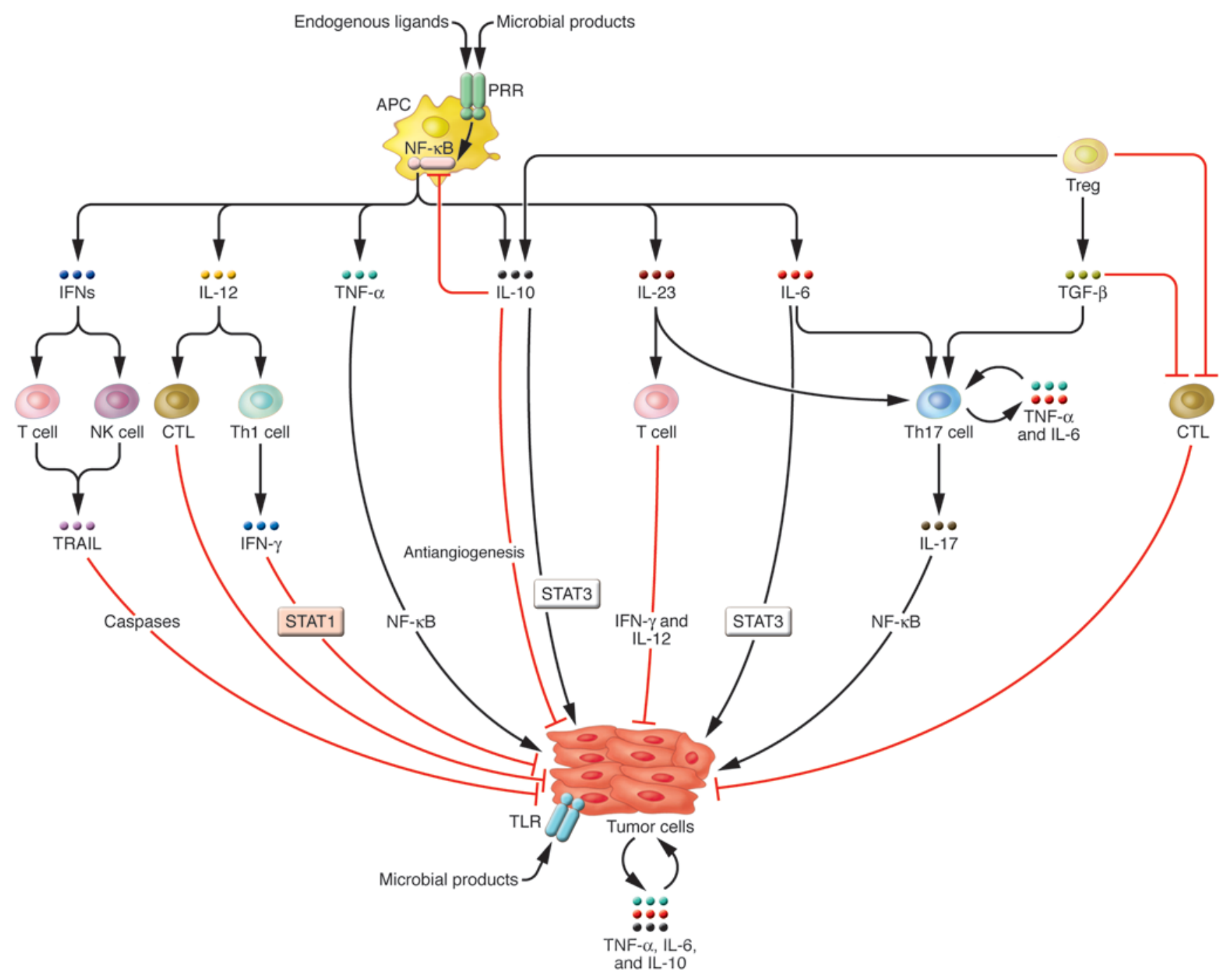

Figure 3

Interactions between various cell types in the tumor microenvironment determine the effects of cytokines on tumor development and progression. Upon pathogen infection, both proinflammatory and antiinflammatory cytokines are produced by activated myeloid cells. In addition to their direct effects on tumor cell growth, survival, and invasive properties, cytokines can govern the functions of Th1 cells, NK cells, Tregs, and Th17 cells, all of which infiltrate the tumor. Treg-mediated suppression of antitumor CTL responses and induction of inflammatory Th17 cell-mediated responses contribute to tumor progression. Paradoxically, IL-10 can mediate the antitumor effects of Tregs. IL-23, TGF- $\beta$, IL- 6 , and TNF- $\alpha$ promote the development of Th17 cells, which have a central role in coordinating chronic inflammatory responses. IL-23 can induce the release of IFN- $\gamma$ and IL-12 from activated T cells, TNF- $\alpha$ and IL-12 from APCs, and IL-17 from Th17 cells. In certain cases, cytokines such as TNF- $\alpha$ and IL-6 might be produced by tumor cells and function in an autocrine and paracrine fashion.

TRAIL is mainly produced by activated T cells and NK cells and is one of the major mediators of antitumor immunity. Unlike TNF- $\alpha$, TRAIL is able to induce apoptosis in various tumor cell types but has only negligible effects on normal cells (Figure 2) (52). TRAIL-deficient mice or mice treated with TRAIL-specific neutralizing antibody exhibit increased susceptibility to experimentally induced and spontaneous tumors (53), suggesting an important role for endogenous TRAIL in tumor surveillance. When $\mathrm{T}$ cells are rendered TRAIL deficient, a much weaker graftversus-tumor effect was observed (54). However, not all tumor cells are TRAIL sensitive, and NF- $\mathrm{KB}$ activation through TNF- $\alpha$ or other pro-survival factors confers on the tumor cells resistance to TRAIL-mediated cytotoxicity (39). Therefore, full real- ization of the antitumor activity of TRAIL requires inhibition of $\mathrm{NF}-\kappa \mathrm{B}$ in the tumor cell or neutralization of NF- $\mathrm{\kappa B}$-activating, tumor-promoting cytokines, such as TNF- $\alpha$.

\section{IL-6}

IL-6 is a potent pleiotropic inflammatory cytokine that is considered a key growth-promoting and antiapoptotic factor (55). The IL- 6 receptor complex is a heterodimer consisting of IL-6R $\alpha$ and glycoprotein 130 (gp130), the latter of which is responsible for signal transduction (56). Activation of gp130 triggers phosphorylation of the STAT proteins STAT1 and STAT3 by JAK1 (Figure 2). STAT3 has a predominant role in IL-6 signal transduction, and its roles in malignant cell proliferation and 
survival have been well documented. By contrast, STAT1 inhibits tumor cell growth (56). JAK-mediated STAT tyrosine phosphorylation allows dimerization, nuclear translocation, and activation of specific target genes (57). Most IL-6 target genes are involved in cell cycle progression and suppression of apoptosis, which underscores the importance of IL-6 in tumorigenesis (58).

IL- 6 is suggested to have a pivotal role in the pathogenesis of Kaposi sarcoma (59) and MM (60). Recent studies also suggest an association between circulating IL- 6 and elevated risk of developing Hodgkin lymphoma (61). Furthermore, a promoter polymorphism study suggests that IL 6 is a predisposing genetic factor that contributes to breast cancer prognosis, with a $\mathrm{G} / \mathrm{C}$ polymorphism within the promoter region of the IL 6 gene that is associated with high levels of IL- 6 production correlating with a worse prognosis (62). Clear evidence that IL-6 governs the growth of MM, a malignant disorder of plasma cells, has come from studies using $I l 6^{-1-}$ mice, which were found to be resistant to plasmacytoma induction (63). In MM, IL-6 is produced by stromal cells in the bone marrow, and its synthesis by these cells can be further enhanced by their interaction with malignant plasma cells (64). Furthermore, in response to infection-activated TLR signaling, MM cells also produce IL-6, which promotes their growth in an autocrine manner (41). New IL-6 antagonists are being evaluated for treatment of MM (65).

IBD is associated with high concentrations of IL-6 (32). Antibody-mediated inhibition of IL- 6 signaling retarded development of chemical-induced colitis-associated colon cancer (CAC) (66). CAC development can also be retarded by deleting the gene encoding IKK $\beta$ in myeloid cells, a process that is known to interfere with IL-6 production during early carcinogenesis (67). Importantly, deletion of the gene encoding IKK $\beta$ in myeloid cells and inhibition of IL- 6 signaling decrease tumor size $(66,67)$, suggesting that IL-6 is mostly responsible for stimulation of tumor growth in these models of cancer (Figure 3).

In addition to classic IL-6 signaling, secretion of soluble IL-6 receptor (sIL-6R) can trigger IL-6 trans-signaling and is critically involved in the development of colon cancer (68). In this disease, it has been suggested that shedding of sIL-6R from adenocarcinoma cells contributes to $T$ cell survival and enhances the production of more IL- 6 by T cells (66). Such findings suggest that IL- 6 antagonists might be useful prophylactically or therapeutically for the treatment of humans with CAC. A recent study further demonstrated a novel form of IL- 6 signaling. In MM cells that express high levels of IL-6R $\alpha$, IL-6R $\alpha$ and the IGF1 receptor are recruited to lipid rafts following exposure of the cells to IL-6 (69). This facilitates hetero-oligomerization of both receptors and leads to JAK-independent activation of AKT, presumably via the IGF1 receptor (69). This type of cross-talk might provide a means for JAK-independent IL-6 signaling in tumor cell survival.

\section{IL-17}

Recently, a new T cell subset named "Th17," characterized by the production of IL-17, was identified as an important player in inflammatory responses (Figure 3) (70). The production of IL-17 relies on STAT3 activation triggered by IL-23 (71). IL-17 induces the recruitment of immune cells to peripheral tissues, a response that requires NF- $\mathrm{KB}$ activation after IL-17 receptor engagement (Figure 2) (72, 73). IL-17 also leads to the induction of many proinflammatory factors, including TNF- $\alpha$, IL- 6 , and IL- $1 \beta$, sug- gesting an important role for IL-17 in localizing and amplifying inflammation (74-76). Furthermore, TNF- $\alpha$ and IL-6, which are both produced by Th17 cells, not only support Th17 cell development but also synergize with IL-17 to enhance the production of proinflammatory mediators (76).

Several studies have begun to address the role of IL-17 in chronic inflammation and cancer (72). IL-17-overexpressing human cervical cancer cells and non-small cell lung carcinoma (NSCLC) cells show substantially greater ability to form tumors in immunocompromised mice compared with control cells not overexpressing IL-17 $(77,78)$. Similarly, IL-17 overexpression in fibrosarcoma cell lines enhances their tumorigenic growth in C57BL/6 mice (79). This tumor-promoting effect has mainly been attributed to the proangiogenic activity of $\operatorname{IL}-17(77,78)$. In primary NSCLC samples, IL-17 expression has frequently been detected in tumor-infiltrating inflammatory cells and was associated with increased tumor vascularity (78). However, enhanced cervical cancer growth elicited by IL-17 was associated with increased expression of IL- 6 and macrophage recruitment to the tumor sites (77). Therefore, IL-17 might also function through IL- 6 to promote tumor development. A potential role for IL-17 in promoting human cervical cancer is suggested by its frequent expression in patients whose tumors show $\mathrm{CD}^{+} \mathrm{T}$ cell infiltration (77).

However, there is also evidence that IL-17 might be involved in tumor surveillance in immunocompetent mice (80). Therefore, current studies of the role of IL-17 in tumor development are still limited. Undoubtedly, it is necessary to determine whether the dominant function of IL-17 is in tumor promotion or tumor surveillance, and critical evaluation in appropriate mouse models of cancer using genetically altered animals lacking specific IL-17 or IL-17 receptor isoforms should address this issue.

\section{IL-12 and IL-23}

IL-12 and IL-23 belong to the IL-12 family of proinflammatory heterodimeric cytokines and are composed of IL-12p40/IL-12p35 and IL-12p40/IL-23p19 subunits, respectively (81). They are mainly produced by activated APCs and accessory cells such as DCs and phagocytes (82). The receptors for these cytokines are also heterodimeric - IL-12 binds an IL-12R $\beta$-IL-12R $\beta 2$ heterodimer, whereas IL-23 binds an IL-12R $\beta 1$-IL-23R heterodimer (Figure 2) (81). The receptors for both IL-12 and IL-23 are mainly expressed on T cells, NK cells, and NKT cells. However, low levels of the receptor for IL-23 are also expressed on monocytes, macrophages, and DCs (82). Both cytokines activate TYK2 and JAK2 as well as STAT1, STAT3, STAT4, and STAT5 (81). Although IL-12 activates STAT4 most efficiently, IL-23 preferentially activates STAT3 (Figure 2) (81). Despite the similarities in receptor subunit and signaling, recent studies have shown that IL-12 and IL-23 drive divergent immunological pathways and exert distinct effects on tumor development.

Endogenous IL-12 is important for host resistance to tumors; the antitumor activity of IL-12 has been extensively reported in mouse models of cancer, where it has been shown to inhibit tumorigenesis and induce regression of established tumors (82). The major antitumor activities of IL-12 rely on its ability to promote Th1 adaptive immunity and CTL responses (Figure 3) (82). IFN- $\gamma$ produced by naive Th cells also contributes to the antitumor activity of IL-12. IFN- $\gamma$ has both a direct toxic effect on cancer cells and antiangiogenic activity (82). The use of IL-12 
in cancer therapy, however, is hindered by severe toxic side effects, primarily because of the extremely high levels of IFN- $\gamma$ that it induces (82).

Although IL-23 can enhance the proliferation of memory T cells and the production of IFN- $\gamma$ and IL-12 by activated T cells (83), it can also drive Th17-mediated responses, induce IL-17 production, and promote end-stage inflammation (Figure 3) (71). Therefore, IL-23 can exert effects similar to those of IL-17. Moreover, IL-23 might function in an autocrine manner to induce the production of TNF- $\alpha$ by macrophages and IL-12 by DCs (83). Immunization of Il23p19-/- mice with collagen results in fewer IL-17-producing T cells and limits the upregulation of IL- 6 and TNF- $\alpha$ (84). Animal studies have so far revealed contradictory effects of IL-23 on tumor growth (85-87). Resistance to skin tumor formation in response to chemical carcinogenesis was observed in Il23p19-/- mice (85). Moreover, the growth of transplanted tumors was restricted in hosts deficient in IL-23 or IL-23R (85). In this study, it was shown that, along with having an effect on tumor growth, IL-23 signaling results in upregulation of MMP9, increased angiogenesis, and decreased recruitment of $\mathrm{CD}^{+} \mathrm{T}$ cells to tumors. Therefore, IL-23-mediated inflammatory processes might provide a tumorpromoting microenvironment (85). However, in other studies, mice inoculated with IL-23-transduced tumor cells displayed increased tumor rejection, but this effect was only observed at very late time points after tumor inoculation $(86,87)$; $\mathrm{CD}^{+} \mathrm{T}$ cells had an important role in this IL-23-mediated antitumor activity (87).

\section{IL-10}

Another cytokine that activates STAT3 is IL-10 (Figure 2) (57). However, the effects of IL-10 are dramatically opposed to those of IL-6, as IL-10 is immunosuppressive and antiinflammatory (88). IL-10 inhibits NF- $\mathrm{KB}$ activation through ill-defined mechanisms $(89,90)$ and consequently inhibits the production of proinflammatory cytokines, including TNF- $\alpha$, IL-6, and IL-12 (91). Given this, it is no wonder that IL-10 inhibits tumor development and progression (Figure 3 ). The most striking effects of IL-10 are seen in $\mathrm{Il10}^{-/-}$mice, which are more prone to colonic inflammation and CAC when chronically infected with certain enteric bacteria, such as Helicobacter hepaticus $(92,93)$. When newborn $\mathrm{Il10}^{-/-}$mice were treated with exogenous IL-10, they failed to develop any signs of intestinal inflammation or CAC (92).

Recent studies emphasize an essential link between IL-10dependent antitumor activity and $\mathrm{CD} 4^{+} \mathrm{CD} 25^{+}$Tregs (Figure 3) (94-97). In mice lacking RAG2, which lack functional lymphocytes, infection with $H$. hepaticus leads to colonic inflammation and adenocarcinoma, whereas infection of wild-type mice does not lead to these pathologies, suggesting that lymphocytes are required for preventing colonic inflammation (94). Accordingly, adoptive transfer of wild-type Tregs into Rag2-/- hosts prevents $H$. hepaticus-induced colon cancer $(94,95)$. A similar adoptive

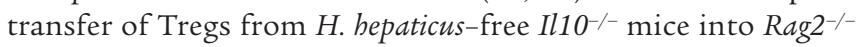
hosts demonstrated that IL-10 released by Tregs is needed for maintaining homeostasis of mucosal immune responses and for inhibition of IBD, dysplasia, and colon cancer $(95,96)$.

The IL-10-mediated antitumor activity of Tregs has also been observed in $\mathrm{ApC}^{\mathrm{Min} / \mathrm{+}}$ mice, which have a germline multiple intestinal neoplasia (Min) mutation in one of their adenomatosis polyposis coli tumor suppressor genes and therefore develop intestinal adenomas (97). Transfer of wild-type Tregs into $A p c^{\mathrm{Min} / \mathrm{H}}$ mice prevents the development of adenomas and induces the rapid regression of established tumors, whereas transfer of $I l 10^{-/-}$Tregs fails to exert such effects (97). Decreased TNF- $\alpha$ and IFN- $\gamma$ expression in mice receiving wildtype Tregs was also noticed (97). Glioma-specific $\mathrm{CD}^{+} \mathrm{T}$ cells have also been shown to require IL-10 for antitumor activity (98), and in xenograft studies, expression of IL-10 in melanoma or mammary or ovarian carcinomas resulted in antitumor effects $(99,100)$.

The mechanisms responsible for IL-10 inhibition of colitis are not completely clear but might be linked to its ability to counteract IL-12-driven inflammation $(95,101)$ or its ability to inhibit NF-кB activation (Figure 3$)(89,90)$. Indeed, enhanced IL-12p40 production by immune cells is a key feature of colonic inflammation in Il10 ${ }^{-/}$mice (101), and absence of IL-10-induced STAT3 activation was suggested to enhance NF- $\mathrm{KB}$ recruitment to the $I l 12 p 40$ promoter (90). Suppression of TNF- $\alpha$ and IL-12 release by DCs and macrophages might also contribute to the antitumor activity of Tregs and IL-10 (102). However, it is not clear how STAT3 activation by IL-10 results in an antitumor effect, whereas STAT3 activation by IL- 6 is considered to be pro-tumorigenic. More recent studies also suggest that IL-10 possesses immunostimulatory activity that enhances antitumor immunity $(103,104)$.

IL-10 has also been shown to modulate apoptosis and suppress angiogenesis during tumor regression $(105,106)$. Expression of IL-10 in mammary and ovarian carcinoma xenografts inhibits tumor growth and spread $(100,105)$. One mechanism by which IL-10 inhibits tumor growth was suggested to depend on downregulation of $\mathrm{MHC}$ class I expression, leading to enhanced NK cell-mediated tumor cell lysis (105). Inhibition of the tumor stroma was suggested to contribute to the antiangiogenic activity of IL-10 (106). The ability of IL-10 to downregulate VEGF, TNF- $\alpha$, and IL- 6 production by TAMs might also account for its inhibitory effect on the tumor stroma (99).

Although IL-10 usually exerts antitumor activity, its biological effects are not all that simple, and consistent with its ability to activate STAT3, it might also promote tumor development (Figure 2). Direct effects of IL-10 on tumor cells that might favor tumor growth have been reported. For example, an IL-10 autocrine and/or paracrine loop might have an important role in tumor cell proliferation and survival (107). The basis for this effect is primarily STAT3 activation, leading to upregulation of antiapoptotic genes such as BCL-2 or BCL- $\mathrm{X}_{\mathrm{L}}(107,108)$. In addition, expression of IL-10 by tumor cells and TAMs is thought to promote the development of Burkitt lymphoma through the production of the TNF family member BAFF, which promotes B cell and lymphoma survival (109). An elevated amount of IL-10 in the plasma has been correlated with poor prognosis in diffuse large B cell lymphoma patients (110). A role for IL-10 in the progression of B cell malignancies is also seen in $\mathrm{Il10}^{-/-}$mice, in which B cell tumors grow more slowly (111). In a B16-melanoma xenograft model, IL-10-transfected cancer cells form more vascularized tumors and exhibit further growth (112). In addition to direct growth modulation of cancer cells, the ability of IL-10 to suppress adaptive immune responses has also been suggested to favor tumor escape from immune surveillance (104).

In summary, IL-10 has complex effects on tumor development. In many experimental systems, IL-10 is found to exert antitumor activity, but in other cases it can be pro-tumorigenic. These dramatically opposing effects of IL-10 might depend on interactions with either cytokines or factors found in the tumor microenvironment, as it is unlikely that IL-10 functions in isolation. A better understanding of IL-10 signaling is needed before its effects on tumor growth and antitumor immunity can be fully explained. 


\section{TGF- $\beta$}

TGF- $\beta$, like IL-10, is not only a powerful pleiotropic immunosuppressive and antiinflammatory cytokine but also a central regulator in Treg proliferation and function (Figure 3) (113-115). TGF- $\beta$ signals mainly through activation of SMAD transcription factors, but it also leads to MAPK activation (Figure 2) $(116,117)$. Defective TGF- $\beta$ signaling due to mutational inactivation of the type 2 TGF- $\beta$ receptor (T $\beta$ RII) has been found to occur frequently in human colon cancer $(118,119)$, where TGF- $\beta$ potently inhibits the growth of colon epithelial cells (117). Such mutations occur at the adenoma-to-carcinoma transition or at a later stage, indicating that the tumor suppressor effect of TGF- $\beta$ is mainly critical at late stages of colon carcinogenesis (120). In addition to direct tumor suppressor activity on colon epithelial cells and antiinflammatory effects on T cells, TGF- $\beta$ has been implicated in Treg-mediated suppressive activity (114). SMAD3 is a key mediator of the antiinflammatory and immunosuppressive activities of TGF- $\beta$ in the colon (121). Accordingly, both TGF- $\beta 1$ - and SMAD3-deficient mice exhibit increased colon carcinogenesis that depends on the presence of certain enteric bacteria, possibly $H$. hepaticus $(122,123)$; germ-free Tgfb1 $1^{-/-}$and Smad3 $3^{-/-}$mice do not develop colon cancer when $H$. hepaticus is no longer present $(122,123)$. Interestingly, TGF- $\beta$ signaling prevents the release of IL- 6 from Th1 cells during the late stages of CAC and therefore functions to control tumor growth (66). Conversely, IL-6-activated STAT3 signaling counteracts the TGF- $\beta$-mediated cytostatic effect through induction of inhibitory SMAD7 (124).

Despite its pronounced antiinflammatory activity and growth inhibition of early tumor cells, TGF- $\beta$ might also enhance tumor progression. Carcinomas often secrete excess TGF- $\beta$ and respond to it by enhanced epithelial-to-mesenchymal transition, tissue invasion, and metastasis (117). Such invasive action of TGF- $\beta$ has been well documented in mouse models of skin carcinomas (117). In addition, both TGF- $\beta$-induced changes in the microenvironment, to favor angiogenesis, and inhibition of tumor-specific $\mathrm{CD}^{+} \mathrm{T}$ cells promote tumor development (Figure 3$)(117,125)$. In summary, the complex role of TGF- $\beta$ in tumor suppression and progression might be stage and cancer cell type dependent.

\section{Conclusions}

The evidence reviewed in this Review demonstrates that activation of innate immunity and inflammation results in the production of cytokines that can either stimulate or inhibit tumor growth and progression. By and large, most proinflammatory cytokines produced by either host immune cells or tumor cells themselves promote tumor development. By contrast, proapoptotic (TRAIL) and antiinflammatory (IL-10 and TGF- $\beta$ ) cytokines usually interfere with tumor development. These findings provide a unique therapeutic opportunity based on selective interference with the action of proinflammatory and tumor-promoting cytokines while enhancing the activity of proapoptotic and antiinflammatory cytokines. In addition to selective modulation of cytokine signaling, interfering with NF- $\mathrm{\kappa B}$ activation in tumor cells can further prevent the pro-survival and growth-promoting effects of proinflammatory cytokines such as TNF- $\alpha$ and render the cancer cells more susceptible to elimination by proapoptotic cytokines such as TRAIL.

Address correspondence to: Michael Karin, University of California, San Diego, 9500 Gilman Drive \#0723, La Jolla, California 92093-0723, USA. Phone: (858) 534-1361; Fax: (858) 534-8158; E-mail: karinoffice@ucsd.edu.
1. Hanahan, D., and Weinberg, R.A. 2000. The hallmarks of cancer. Cell. 100:57-70.

2. Coussens, L.M., and Werb, Z. 2002. Inflammation and cancer. Nature. 420:860-867.

3. Shacter, E., and Weitzman, S.A. 2002. Chronic inflammation and cancer. Oncology. 16:217-226.

4. Hussain, S.P., Hofseth, L.J., and Harris, C.C. 2003. Radical causes of cancer. Nat. Rev. Cancer. 3:276-285.

5. Fox, J.G., and Wang, T.C. 2007. Inflammation, atrophy, and gastric cancer. J. Clin. Invest. 117:60-69. doi:10.1172/JCI30111.

6. Dobrovolskaia, M.A., and Kozlov, S.V. 2005 Inflammation and cancer: when NF-kappaB amalgamates the perilous partnership. Curr. Cancer Drug Targets. 5:325-344.

7. de Visser, K.E., Eichten, A., and Coussens, L.M 2006. Paradoxical roles of the immune system during cancer development. Nat. Rev. Cancer. 6:24-37.

8. Balkwill, F., Charles, K.A., and Mantovani, A. 2005. Smoldering and polarized inflammation in the initiation and promotion of malignant disease. Cancer Cell. 7:211-217.

9. Karin, M., and Greten, F.R. 2005. NF-кB: linking inflammation and immunity to cancer development and progression. Nat. Rev. Immunol. 5:749-759.

10. Karin, M. 2006. Nuclear factor- $\kappa B$ in cancer development and progression. Nature. 441:431-436.

11. Luster, A.D., Alon, R., and von Andrian, U.H. 2005. Immune cell migration in inflammation: present and future therapeutic targets. Nat. Immunol. 6:1182-1190

12. Balkwill, F., and Mantovani, A. 2001. Inflammation and cancer: back to Virchow? Lancet. 357:539-545.

13. Mantovani, A., Sozzani, S., Locati, M., Allavena, P., and Sica, A. 2002. Macrophage polarization: tumor-associated macrophages as a paradigm for polarized M2 mononuclear phagocytes. Trends Immunol. 23:549-555.

14. Ben-Baruch, A. 2006. Inflammation-associated immune suppression in cancer: the roles played by cytokines, chemokines and additional mediators. Semin. Cancer Biol. 16:38-52.

15. Smyth, M.J., Cretney, E., Kershaw, M.H., and Hayakawa, Y. 2004. Cytokines in cancer immunity and immunotherapy. Immunol. Rev. 202:275-293.

16. Kim, R., Emi, M., Tanabe, K., and Arihiro, K. 2006. Tumor-driven evolution of immunosuppressive networks during malignant progression. Cancer Res. 66:5527-5536.

17. Hadden, J.W. 2003. Immunodeficiency and cancer: prospects for correction. Int. Immunopharmacol. 3:1061-1071.

18. Luo, J.L., Kamata, H., and Karin, M. 2005. IKK/ NF- $\mathrm{KB}$ signaling: balancing life and death-a new approach to cancer therapy. J. Clin. Invest. 115:2625-2632. doi:10.1172/JCI26322.

19. Karin, M., Cao, Y., Greten, F.R., and Li, Z.W. 2002. $\mathrm{NF}-\kappa \mathrm{B}$ in cancer: from innocent bystander to major culprit. Nat. Rev. Cancer. 2:301-310.

20. Karin, M. 2006. NF-кB and cancer: mechanisms and targets. Mol. Carcinog. 45:355-361.

21. Pidgeon, G.P., et al. 1999. The role of endotoxin/ lipopolysaccharide in surgically induced tumour growth in a murine model of metastatic disease. Br. J. Cancer. 81:1311-1317.

22. Taketomi, A., et al. 1997. Circulating intercellular adhesion molecule- 1 in patients with hepatocellular carcinoma before and after hepatic resection. Hepatogastroenterology. 44:477-483.

23. Medzhitov, R. 2001. Toll-like receptors and innate immunity. Nat. Rev. Immunol. 1:135-145.

24. Akira, S., Uematsu, S., and Takeuchi, O. 2006.
Pathogen recognition and innate immunity. Cell. 124:783-801.

25. Fritz, J.H., Ferrero, R.L., Philpott, D.J., and Girardin, S.E. 2006. Nod-like proteins in immunity, inflammation and disease. Nat. Immunol. 7:1250-1257.

26. Robinson, M.J., Sancho, D., Slack, E.C., Leibundgut-Landmann, S. and Sousa, C.R. 2006. Myeloid C-type lectins in innate immunity. Nat. Immunol. 7:1258-1265.

27. Klesney-Tait, J., Turnbull, I.R., and Colonna, M. 2006. The TREM receptor family and signal integration. Nat. Immunol. 7:1266-1273.

28. Han, J., and Ulevitch, R.J. 2005. Limiting inflammatory responses during activation of innate immunity. Nat. Immunol. 6:1182-1189.

29. Staib, F., et al. 2005. The p53 tumor suppressor network is a key responder to microenvironmental components of chronic inflammatory stress. Cancer Res. 65:10255-10264.

30. Sun, J., et al. 2006. Interactions of sequence variants in interleukin-1 receptor-associated kinase 4 and the toll-like receptor 6-1-10 gene cluster increase prostate cancer risk. Cancer Epidemiol. Biomarkers Prev. 15:480-485.

31. Hugot, J.P., et al. 2001. Association of NOD2 leucine-rich repeat variants with susceptibility to Crohn's disease. Nature. 411:599-603.

32. Podolsky, D.K. 2002. Inflammatory bowel disease. N. Engl. J. Med. 347:417-429.

33. Eckmann, L., and Karin, M. 2005. NOD2 and Crohn's disease: Loss or gain of function? Immunity. 22:661-667.

34. Sehouli, J., Mustea, A., Konsgen, D., Katsares, I., and Lichtenegger, W. 2002. Polymorphism of IL-1 receptor antagonist gene: role in cancer. Anticancer Res. 22:3421-3424. 
35. Troost, E., et al. 2003. The role of interleukin1 beta and other potential genetic markers as indicators of gastric cancer risk. Can. J. Gastroenterol. 17(Suppl. B):8B-12B

36. Apte, R.N., et al. 2006. The involvement of IL-1 in tumorigenesis, tumor invasiveness, metastasis and tumor-host interactions. Cancer Metastasis Rev. 25:387-408.

37. Lewis, A.M., Varghese, S., Xu, H., and Alexander, H.R. 2006. Interleukin-1 and cancer progression: the emerging role of interleukin-1 receptor antagonist as a novel therapeutic agent in cancer treatment. J. Transl. Med. 4:48.

38. Harmey, J.H., et al. 2002. Lipopolysaccharideinduced metastatic growth is associated with increased angiogenesis, vascular permeability and tumor cell invasion. Int. J. Cancer. 101:415-422.

39. Luo, J.L., Maeda, S., Hsu, L.C., Yagita, H., and Karin, M. 2004. Inhibition of NF-KB in cancer cells converts inflammation-induced tumor growth mediated by TNF- $\alpha$ to TRAIL-mediated tumor regression. Cancer Cell. 6:297-305.

40. Almasan, A., and Ashkenazi, A. 2003. Apo2L/ TRAIL: apoptosis signaling, biology, and potential for cancer therapy. Cytokine Growth Factor Rev 14:337-348

41. Jego, G., et al. 2006. Pathogen-associated molecular patterns are growth and survival factors for human myeloma cells through Toll-like receptors. Leukemia. 20:1130-1137.

42. Bohnhorst, J., et al. 2006. Toll-like receptors mediate proliferation and survival of multiple myeloma cells. Lenkemia. 20:1138-1144.

43. Lin, W.J., and Yeh, W.C. 2005. Implication of Tolllike receptor and tumor necrosis factor alpha signaling in septic shock. Shock. 24:206-209.

44. Mocellin, S., Rossi, C.R., Pilati, P., and Nitti, D. 2005. Tumor necrosis factor, cancer and anticancer therapy. Cytokine Growth Factor Rev. 16:35-53.

45. Yang, H., et al. 2006. TNF- $\alpha$ inhibits asbestosinduced cytotoxicity via a NF- $\mathrm{KB}$-dependent pathway, a possible mechanism for asbestosinduced oncogenesis. Proc. Natl. Acad. Sci. U. S. A. 103:10397-10402.

46. Elgert, K.D., Alleva, D.G., and Mullins, D. W. 1998. Tumor-induced immune dysfunction: the macrophage connection. J. Leukoc. Biol. 64:275-290.

47. Moore, R.J., et al. 1999. Mice deficient in tumor necrosis factor-alpha are resistant to skin carcinogenesis. Nat. Med. 5:828-831.

48. Arnott, C.H., et al. 2004. Expression of both TNF- $\alpha$ receptor subtypes is essential for optimal skin tumour development. Oncogene. 23:1902-1910.

49. Pikarsky, E., et al. 2004. NF-кB functions as a tumour promoter in inflammation-associated cancer. Nature. 431:461-466.

50. Knight, B., et al. 2000. Impaired preneoplastic changes and liver tumor formation in tumor necrosis factor receptor type 1 knockout mice. J. Exp. Med. 192:1809-1818.

51. Kitakata, H., et al. 2002. Essential roles of tumor necrosis factor receptor p55 in liver metastasis of intrasplenic administration of colon 26 cells. Cancer Res. 62:6682-6687.

52. LeBlanc, H.N., and Ashkenazi, A. 2003. Apo2L/ TRAIL and its death and decoy receptors. Cell Death Differ. 10:66-75.

53. Takeda, K., et al. 2002. Critical role for tumor necrosis factor-related apoptosis-inducing ligand in immune surveillance against tumor development. J. Exp. Med. 195:161-169.

54. Schmaltz, C., et al. 2002. T cells require TRAIL for optimal graft-versus tumor activity. Nat. Med. 8:1433-1437.

55. Ishihara, K., and Hirano, T. 2002. IL-6 in autoimmune disease and chronic inflammatory proliferative disease. Cytokine Growth Factor Rev. 13:357-368.

56. Hodge, D.R., Hurt, E.M., and Farrar, W.L. 2005.
The role of IL- 6 and STAT3 in inflammation and cancer. Eur. J. Cancer. 41:2502-2512.

57. O'Shea, J.J., Gadina, M., and Schreiber, R.D. 2002. Cytokine signaling in 2002: new surprises in the Jak/Stat pathway. Cell. 109(Suppl.):S121-S131.

58. Haura, E.B., Turkson, J., and Jove, R. 2005. Mechanisms of disease: insights into the emerging role of signal transducers and activators of transcription in cancer. Nat. Clin. Pract. Oncol. 2:315-324.

59. Osborne, J., Moore, P.S., and Chang, Y. 1999. KSHVencoded viral IL- 6 activates multiple human IL-6 signalling pathways. Hum. Immunol. 60:921-927.

60. Bommert, K., Bargou, R.C., and Stuhmer, T. 2006. Signalling and survival pathways in multiple myeloma. Eur. J. Cancer. 42:1574-1580.

61. Cozen, W., et al. 2004. IL-6 levels and genotype are associated with risk of young adult Hodgkin lymphoma. Blood. 103:3216-3221.

62. Berger, F.G. 2004. The interleukin- 6 gene: a susceptibility factor that may contribute to racial and ethnic disparities in breast cancer mortality. Breast Cancer Res. Treat. 88:281-285.

63. Gado, K., et al. 2001. Mouse plasmacytoma: an experimental model of human multiple myeloma. Haematologica. 86:227-236.

64. Chauhan, D., et al. 1996. Multiple myeloma cell adhesion-induced interleukin- 6 expression in bone marrow stromal cells involves activation of NF- $\mathrm{KB}$. Blood. 87:1104-1112.

65. Feng, J., et al. 2006. The rational designed antagonist derived from the complex structure of interleukin- 6 and its receptor affectively blocking interleukin- 6 might be a promising treatment in multiple myeloma. Biochimie. 88:1265-1273.

66. Becker, C., et al. 2004. TGF $\beta$ suppresses tumor progression in colon cancer by inhibition of IL- 6 trans-signaling. Immunity. 21:491-501.

67. Greten, F.R., et al. 2004. IKK $\beta$ links inflammation and tumorigenesis in a mouse model of colitisassociated cancer. Cell. 118:285-296.

68. Rose-John, S., Scheller, J., Elson, G., and Jones, S.A 2006. Interleukin- 6 biology is coordinated by membrane-bound and soluble receptors: role in inflammation and cancer. J. Leukoc. Biol. 80:227-236.

69. Abroun, S., et al. 2004. Receptor synergy of interleukin-6 (IL-6) and insulin-like growth factor-I in myeloma cells that highly express IL- 6 receptor $\alpha$. Blood. 103:2291-2298.

70. Mangan, P.R., et al. 2006. Transforming growth factor- $\beta$ induces development of the $T_{H} 17$ lineage. Nature. 441:231-234.

71. Cho, M.L., et al. 2006. STAT3 and NF-אB signal pathway is required for IL-23-mediated IL-17 production in spontaneous arthritis animal model IL-1 receptor antagonist-deficient mice. J. Immunol. 176:5652-5661.

72. Moseley, T.A., Haudenschild, D.R., Rose, L., and Reddi, A.H. 2003. Interleukin-17 family and IL-17 receptors. Cytokine Growth Factor Rev. 14:155-174.

73. Witowski, J., Ksiazek, K., and Jorres, A. 2004. Interleukin-17: a mediator of inflammatory responses. Cell Mol. Life Sci. 61:567-579.

74. Langrish, C.L., et al. 2005. IL-23 drives a pathogenic $T$ cell population that induces autoimmune inflammation. J. Exp. Med. 201:233-240.

75. Park, H., et al. 2005. A distinct lineage of CD4 T cells regulates tissue inflammation by producing interleukin 17. Nat. Immunol. 6:1133-1141.

76. Ruddy, M.J., et al. 2004. Functional cooperation between interleukin-17 and tumor necrosis factor- $\alpha$ is mediated by CCAAT/enhancer-binding protein family members. J. Biol. Chem. 279:2559-2567.

77. Tartour, E., et al. 1999. Interleukin 17, a T-cellderived cytokine, promotes tumorigenicity of human cervical tumors in nude mice. Cancer Res. 59:3698-3704.

78. Numasaki, M., et al. 2005. IL-17 enhances the net angiogenic activity and in vivo growth of human non-small cell lung cancer in SCID mice through promoting CXCR-2-dependent angiogenesis. J. Immunol. 175:6177-6189.

79. Numasaki, M., et al. 2003. Interleukin-17 promotes angiogenesis and tumor growth. Blood. 101:2620-2727.

80. Benchetrit, F., et al. 2002. Interleukin-17 inhibits tumor cell growth by means of a T-cell-dependent mechanism. Blood. 99:2114-2121.

81. Watford, W.T., et al. 2004. Signaling by IL-12 and IL-23 and the immunoregulatory roles of STAT4. Immunol. Rev. 202:139-156.

82. Trinchieri, G. 2003. Interleukin-12 and the regulation of innate resistance and adaptive immunity. Nat. Rev. 3:133-146.

83. Hao, J.S., and Shan, B.E. 2006. Immune enhancement and anti-tumour activity of IL-23. Cancer Immunol. Immunother. 55:1426-1431.

84. Murphy, C.A., et al. 2003. Divergent pro- and antiinflammatory roles for IL-23 and IL-12 in joint autoimmune inflammation. J. Exp. Med. 198:1951-1957.

85. Langowski, J.L., et al. 2006. IL-23 promotes tumour incidence and growth. Nature. 442:461-465.

86. Ugai, S., et al. 2003. Transduction of the IL-21 and IL-23 genes in human pancreatic carcinoma cells produces natural killer cell-dependent and -independent antitumor effects. Cancer Gene Ther. 10:771-778.

87. Lo, C.H., et al. 2003. Antitumour and antimetastatic activity of IL-23. J. Immunol. 171:600-607

88. Pestka, S., et al. 2004. Interleukin-10 and related cytokines and receptors. Annu. Rev. Immunol. 22:929-979.

89. Schottelius, A.J., Mayo, M.W., Sartor, R.B., and Baldwin, A.S., Jr. 1999. Interleukin-10 signaling blocks inhibitor of kappaB kinase activity and nuclear factor kappaB DNA binding. J. Biol. Chem. 274:31868-31874.

90. Hoentjen, F., Sartor, R.B., Ozaki, M., and Jobin, C. 2005. STAT3 regulates NF- $\kappa B$ recruitment to the IL-12p40 promoter in dendritic cells. Blood. 105:689-696.

91. Moore, K.W., et al. 2001. Interleukin-10 and the interleukin-10 receptor. Annu. Rev. Immunol. 19:683-765.

92. Berg, D.J., et al. 1996. Enterocolitis and colon cancer in interleukin-10-deficient mice are associated with aberrant cytokine production and $\mathrm{CD} 4^{+} \mathrm{TH} 1-$ like responses. J. Clin. Invest. 98:1010-1020.

93. Sellon, R.K., et al. 1998. Resident enteric bacteria are necessary for development of spontaneous colitis and immune system activation in interleukin10-deficient mice. Infect. Immun. 66:5224-5231.

94. Erdman, S.E., et al. 2003. $\mathrm{CD}^{+} \mathrm{CD} 25^{+}$regulatory $\mathrm{T}$ lymphocytes inhibit microbially-induced colon cancer in Rag2-deficient mice. Am. J. Pathol. 162:691-702.

95. Maloy, K., et al. 2003. CD4 ${ }^{+} \mathrm{CD} 25^{+} \mathrm{Tr}$ cells suppress innate immune pathology through cytokinedependent mechanisms. J. Exp. Med. 197:111-119.

96. Erdman, S.E., et al. 2003 . CD4 ${ }^{+} \mathrm{CD} 25^{+}$regulatory lymphocytes require interleukin 10 to interrupt colon carcinogenesis in mice. Cancer Res. 63:6042-6050.

97. Erdman, S.E., et al. 2005. $\mathrm{CD} 4{ }^{+} \mathrm{CD} 25^{+}$regulatory lymphocytes induce regression of intestinal tumors in $\mathrm{Apc}^{\mathrm{Min} /+}$ mice. Cancer Res. 65:3998-4004.

98. Segal, B.M., Glass, D.D., and Shevach, E.M. 2002. Cutting edge: IL-10-producing $\mathrm{CD} 4^{+} \mathrm{T}$ cells mediate tumor rejection. J. Immunol. 168:1-4.

99. Huang, S., Ullrich, S.E., and Bar-Eli, M. 1999. Regulation of tumor growth and metastasis by interleukin-10: the melanoma experience. J. Interferon Cytokine Res. 19:697-703.

100.Kohno, T., et al. 2003. Interleukin-10-mediated inhibition of angiogenesis and tumor growth in mice bearing VEGF-producing ovarian cancer. Cancer Res. 63:5091-5094.

101.Leach, M.W., and Rennick, D.M. 1998. IL-12, but not IFNgamma, plays a major role in sustaining 
the chronic phase of colitis in IL-10-deficient mice. J. Immunol. 161:3143-3149.

102.Larmonier, N, et al. 2007. Tumor-derived $\mathrm{CD} 4{ }^{+} \mathrm{CD} 25^{+}$regulatory $\mathrm{T}$ cell suppression of dendritic cell function involves TGF-beta and IL-10. Cancer Immunol. Immunother. 56:48-59.

103.Vicari, A.P., and Trinchieri, G. 2004. Interleukin-10 in viral diseases and cancer: exiting the labyrinth? Immunol. Rev. 202:223-236.

104.Mocellin, S., Marincola, F.M., and Young, H.A. 2005. Interleukin-10 and the immune response against cancer: a counterpoint. J. Leukoc. Biol. 78:1043-1051.

105.Kundu, N., and Fulton, A.M. 1997. Interleukin10 inhibits tumor metastasis, down-regulates MHC class I, and enhances NK lysis. Cell. Immunol. 180:55-61.

106. Blankenstein, T. 2005. The role of tumor stroma in the interaction between tumor and immune system. Curr. Opin. Immunol. 17:180-186.

107. Sredni, B., et al. 2004. Ammonium trichloro(dioxoethylene-o,o')tellurate (AS101) sensitizes tumors to chemotherapy by inhibiting the tumor interleukin 10 autocrine loop. Cancer Res. 64:1843-1852.

108. Alas, S., et al. 2001. Inhibition of interleukin-10 by rituximab results in down-regulation of bcl-2 and sensitization of B-cell non-Hodgkin's lymphoma to apoptosis. Clin. Cancer Res. 7:709-723.

109.Ogden, C.A., et al. 2005. Enhanced apoptotic cell clearance capacity and B cell survival factor production by IL-10-activated macrophages: implications for Burkitt's lymphoma. J. Immunol. 174:3015-3023.

110.Lech-Maranda, E., et al. 2006. Elevated IL-10 plasma levels correlate with poor prognosis in diffuse large B-cell lymphoma. Eur. Cytokine Netw. 17:60-66.

111. Czarneski, J, et al. 2004. Studies in NZB IL-10 knockout mice of the requirement of IL-10 for progression of B-cell lymphoma. Leukemia. 18:597-606.

112.Garcia-Hernandez, M.L., et al. 2002. Interleukin-10 promotes B16-melanoma growth by inhibition of macrophage functions and induction of tumour and vascular cell proliferation. Immunology. 105:231-243

113. Chen, W.J., and Wahl, S.M. 2003. TGF- $\beta$ : the missing link in $\mathrm{CD} 4{ }^{+} \mathrm{CD} 25^{+}$regulatory $\mathrm{T}$ cell-mediated immunosuppression. Cytokine Growth Factor Rev. 14:85-89.

114.Becker, C., Fantini, M.C., and Neurath, M.F. 2006. TGF- $\beta$ as a T cell regulator in colitis and colon cancer. Cytokine Growth Factor Rev. 17:97-106.

115.Ghiringhelli, F., et al. 2005. Tumor cells convert immature myeloid dendritic cells into TGF- $\beta$ secreting cells inducing $\mathrm{CD} 4{ }^{+} \mathrm{CD} 25^{+}$regulatory $\mathrm{T}$ cell proliferation. J. Exp. Med. 202:919-929.

116.Derynck, R., and Zhang, Y.E. 2003. Smad-dependent and Smad-independent pathways in TGF- $\beta$ family signaling. Nature. 425:577-584.

117. Derynck, R., Akhurst, R.J., and Balmain, A. 2001. TGF- $\beta$ signaling in tumor suppression and cancer progression. Nat. Genet. 29:117-129.

118.Parsons, R., et al. 1995. Microsatellite instability and mutations of the transforming growth factor type II receptor gene in colorectal cancer. Cancer Res. 55:5548-5550.

119.Kim, S.J., Im, Y.H., Markowitz, S.D., and Bang, Y.J. 2000. Molecular mechanisms of inactivation of TGF- $\beta$ receptors during carcinogenesis. Cytokine Growth Factor Rev. 11:159-168.

120.Markowitz, S., et al. 1995. Inactivation of the type II TGF- $\beta$ receptor in colon cancer cells with microsatellite instability. Science. 268:1336-1338.

121.Yang, X., et al. 1999. Targeted disruption of Smad3 results in impaired mucosal immunity and diminished $\mathrm{T}$ cell responsiveness to TGF- $\beta$. EMBO J. 18:1280-1291.

122.Engle, S.J., et al. 2002. Elimination of colon cancer in germ-free transforming growth factor $\beta 1$-deficient mice. Cancer Res. 62:6362-6366.

123.Maggio-Price, L., et al. 2006. Helicobacter infection is required for inflammation and colon cancer in SMAD3-deficient mice. Cancer Res. 66:828-838.

124.Jenkins, B.J., et al. 2005. Hyperactivation of Stat 3 in gp130 mutant mice promotes gastric hyperproliferation and desensitizes TGF- $\beta$ signaling. Nat. Med. 11:845-852.

125.Chen, M.L., et al. 2005. Regulatory T cells suppress tumor-specific CD8 T cell cytotoxicity through TGF- $\beta$ signals in vivo. Proc. Natl. Acad. Sci. U. S. A. 102:419-424. 